\title{
Hibik ng mga manggagawang Pilipino sa piling tula ni
} Amado Vera Hernandez

\author{
Bacalla, Lita A. $\bowtie$ \\ Cebu Normal University, Philippines (lolita_bacalla@yahoo.com.ph)
}

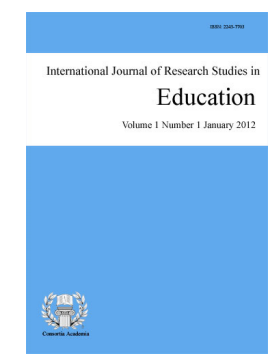

Accepted: 25 October 2020

ISSN: 2243-7703 Online ISSN: 2243-7711

OPEN ACCESS

\section{Abstract}

This paper analyzes the cry of the laborers in the selected poems of Amado Vera Hernandez: "Ang Panday," "Ang Aklasan," "Kung Tuyo na Ang Luha Mo, Aking Bayan," "Ang Buhay," and "Ang Bayani." This study specifically answers the following variables: voice of the persona, poetic vision, addressee and collective complaints. The research uses of qualitative approach using Content Analysis in interpreting and analyzing the meaning embodied in the selected poem. This study is supported by the Formalistic and Mimetic and theories. The following are the findings in the poem of Amado Vera Hernandez: 1)The voice of the persona or the laborer felt discriminated, disappointed, discontented, nationalistic, challenged and hopeful of their situation; 2) the poetic vision: labor contributes to the economic stability of the country, work hard, fight for their right, independent, run the economy of the country, and long for economic progress; 3) Addressee business institutions, exploited laborers, landowner, mining companies, government; 4) collective voice demands for respect of the rights of the laborer with respect to compensation, benefits, hours of works and safety. Based on the findings of the study, the selected poems of Amado Vera Hernandez revealed the cry of the laborer in the society.

Keywords: cry; laborers; persona 


\section{Hibik ng mga manggagawang Pilipino sa piling tula ni Amado Vera Hernandez (Cry of the laborers in the selected poems of Amado Vera Hernandez)}

\section{Introduksyon}

Kasabay sa pag-inog ng buhay, ang bawat nilalang ay kailangang kumayod upang mabuhay. Tungkulin ng tao ang maghanapbuhay katimbang ang sikap at tiyaga upang kumita at magkapera para pangtustos sa mga pangunahing pangangailangan. Sa panahon ngayon, ang mga manggagawang Pilipino ang sobrang naapektuhan sa panahon ng pandemya.Nakalipas na ang isang dekada hanggang sa kasalukuyan, wala pa ring makabuluhang dagdag sahod ang mga manggagawang Pilipino. Humigit daan-libong manggagawa na ang sinibak nitong nakaraang dalawang taon ng pandaigdigang krisis pampinansiyal. Noong Hunyo 2009, 1,663 na manggagawa sa pabrikang Triumph International Philippines Inc. (TIPI) ay tinanggalan ng trabaho dahil umano sa pandaigdigang krisis pang ekonomiya kaya naglunsad sila ng isang kilos-protesta sa Mendiola Bridge noong Agosto 29, 2009.

May mga pag-aaral ni Ortiz (2009), naglahad na mga manggagawang kabataan ay natumbok na nakaranas ng marginalized na sistema sa karapatang bilang batang manggagawa sa lipunan. Ilan lang ito sa mga nagpapatunay na hanggang ngayon marami pa ring mga manggagawang nakakaranas nang hindi pantay na pagtrato. Hindi binigyang- pansin ang kanilang hinanaing at reklamo. Hindi binibigyang-solusyon, sa halip mas isinulong pa ang kontrakwalisasyon na kung saan pwede kang tanggalin kung kailan gusto ng may-ari. Ito'y napakalaking labag sa batas at sa karapatang pantao.

Isang pagsusuri sa mga modernong manggagawa at pagsusuri ng polisiya ng manggagawa sa panlungsod ng Manila (Pante, 2013; San Juan, 2014) na may mga paglabag sa karapatang manggagawa. Ang kanilang pag-aaral ay may kaugnayan sa pag-aaral tungkol sa kalagayan ng manggagawa sa lipunan. Ito ay naglahad sa kalagayan mismo ng mga manggagwa sa lipunan sa pamamagitan na pakikinapanayam samantala ang pag-aaralan na ito na pinagtuanan pansin ang mga kalagayan ng mga manggagawa sa akdang pampanitikan lalo sa genre ng tula. Ayon kay Villafuerte (2003), si Scott ang nagsabi: Ang pag-uugnayan panlipunang ay nakapagdaragdag ng lakas sa manunulat upang ito'y gawing kasangkapan... masusuri at masasalamin ang lipunan”. Hindi na bago sa bansa ang usaping tungkol sa suliranin ng mga manggagawa. Noon pa man, nakakaranas na sila ng paghihirap. Tulad ni Amado Vera Hernandez, naging tulay niya sa paghahatid ang tula upang maipahayag sa mga mambabasa ang mga pangarap at daing ng mga manggagawang Pilipino. Sapagkat ang panitikan ay salamin at kaluluwa ng bansa, sinasalamin dito ang mga pangarap, kaalaman, kaugalian at totoong mukha ng lipunan. Nailalarawan ng manunulat ang uri ng lipunang kanyang ginagalawan sa pamamagitan ng pagbibigay-buhay sa kanyang nakikita't nadarama. May kakaiba siyang lakas upang maipadama niya sa mambabasa ang malakas na pulso ng lipunan. Nagsasalaysay ito ng buhay, pamumuhay, lipunan, pamahalaan, pananampalataya at mga karanasang kaugnay ng iba't ibang uri ng damdaming tulad ng pag-ibig, kaligayahan, kalungkutan, pag-asa, pagkapoot, paghihiganti, pagkasuklam, pagkasindak, pagkamuhi at pangamba.

Sa panulaang Filipino inilalarawan ang iba't ibang mukha ng lipunang ating ginagalawan sa pamamagitan ng tula. Binabakas dito ang mga suliranin at solusyon, kasiyahan at kalungkutan, kapangitan at kagandahan, kalinisan at karumihan, nabubusog at nagugutom, mariwasa at naghihirap. At sa mga paglalarawang ito tanging ang mga manggagawa ang tipikal na Pilipinong nakararanas sa ganitong uri ng karanasan. Ito ay may kaugnayan sa natuklasan ng pag-aaral nina Hechanova at Rebadomia (2010), ng obhektibong kalagayan ng mga manggagawa sa asyenda ng tubuhan, nagkaroon ng konkretong halimbawa para makita ang tunay na kondisyon ng mga manggagawang bukid na nalulugmok sa kahirapan ng buhay dahil sa pananamantala ng iilang naghaharing uri sa lipunan.

Ang makatang si Amado Vera Hernandez ay nagbibigay-larawan sa mga manggagawang Pilipino sa 
pamamagitan ng kanyang nabubuong tula. Bilang makata, ginamit niya ang tula upang maipapahayag niya ang kanyang nakikita sa lipunan, sa kanyang mundong ginagalawan at sa kakaibang damdamin ng ibang tao. Hindi maitatanggi na patuloy pa rin ang mga masasamang nangyayari sa ating komunidad lalong-lalo na sa pampolitikal at pangkarapatan na aspeto. Si Amado Vera Hernandez ay ipinaglalaban niya ang mga karapatan ng manggagawa na pilit ipinagkakait ng mga taong nasa matas na posisyon, mayaman, makapangyarihan at maimpluwensiya. Ninanais ng mananaliksik, na maipapalitaw ang mga tinig ng mga manggagawa sa pagsusuri at mapukaw ang damdamin ng mga mambabasa at ang tagapamahala na mamulat sa katotohanang pangyayari sa kasalukuyan.

Nakaangkla ang pag-aaral sa teoriyang ng panunuring pampanitikan na Pormalistiko at Mimetiko ni Plato. Ang Teoriyang Pormalistiko ay isang uri ng kritisismong pampanitikan na nagbibigay- diin sa porma ng isang teksto at hindi sa nilalaman nito. Binibigyan nito ng markadong atensyon ang istruktura, istilo at paraang artistiko ng isang akda. Samakatwid, ang pisikal na katangian ng akda ang pinakaubod ng pagdulog na ito kung kaya ang tunguhin ng teoriyang na ang matukoy ang (1) nilalaman, (2) kaanyuan o kayarian at (3) paraan ng pagkakasulat ng akda (Villafuerte, 2000). Ang Teoriyang Mimetiko ang ideya na ang sining ay gumagaya sa katotohanan. Ang tagasuri o mambabasa ang magbibigay kahulugan sa pampanitikang gawa na kopya o gaya sa kalikasan. Ang pagsusuring mimetiko ay nauukol sa kaugnayan ng tula sa kalikasan o sa buong mundo. Ayon kay Plato, ang manunulat ay tinuturing na paksa o pinapaksa sa sulatin kaya sapat na itong katibayan sa nasasabing tunay o katotohanan. Binibigyan buhay ang nais ipaabot ng makata gamit ang kanyang tuwirang mensahe.

\subsection{Layunin ng pag-aaral}

Ang pampanitikang pag-aaral na ito ay sumusuri sa mga tinig ng mga manggagawa sa mga piling tula ni Amado Vera Hernandez. Tinitiyak sa pagsusuring ito ang sumusunod na aspeto sa bawat tula:

- ang tinig ng persona,

- ang patulang pananaw,

- ang pinag-uukulan, at

- ang pinag-isang daing.

\section{Metodolohiya}

Kwalitatibong pamamaraan ang ginamit upang masuri ang nilalaman sa piling tulang ni Amado Vera Hernandez. Ang pangunahing pinagmulan ng mga datos ay ang limang piling tula na "Ang Panday," "Ang Aklasan," "Kung Tuyo na Ang Luha Mo Aking Bayan," “Ang Bayani," at "Ang Buhay." Sa tulong ng Analisis na pangnilalaman o Content Analysis. Ito ay pagsusuri at pagpapakahulugan ng mga pinapahayag na teksto sa isang akdang pampanitikan na pag-aaral. Isa-isang sinuri ang mga linyang nagpapahayag batay sa tiyak na suliran.

\section{Mga resulta at pagtalakay}

Inilahad ng mga datos sa ginawang pagsusuri sa mga piling tula ni Amado Vera Hernandez sa tulong ng talahanayan. Mababasa sa ibaba ang kinalalabasan ng pagsusuri gaya ng; ang tinig ng persona, ang patulang pananaw, ang pinag-uukulan at ang pinag-isang daing sa tulong ng teoriyang mimetiko at pormalistiko. Ito ang nagsisilbing presentasyon ng talahanayan sa suliraning tinutukoy sa pagsusuri sa mga piling tula.

\subsection{Ang tinig ng persona}

Tinig ng Persona sa isang tinig na nangingibabaw ng isang tula. Ito ang unang tuon sa pag-susuri ng mananaliksik. Ang tinig na nangingibabaw sa isang tula gamit ang persona. Matutukoy ang tinig ng persona ayon sa tono na makikita sa tula ng bawat linyang o saknong na susuriin. Ang datos na tumutukoy sa unang tiyak 
Bacalla, L. A.

na suliranin ay makikita sa talahanayang.

\section{Talahanayan 1}

Ang tinig ng persona

\begin{tabular}{ll}
\hline Mga tula & \multicolumn{1}{c}{ Tinig ng persona } \\
\hline Ang Panday & $\begin{array}{l}\text { Optimistiko sa pagnanais na mabago ang kanyang sarili, na mapagmalaki sa kakayahan } \\
\text { ng isang panday, at ninais na matahimik at matiwasay na pamumuhay. May } \\
\text { pagpapakahalaga ng trabaho ng isang panday. }\end{array}$
\end{tabular}

Ang Aklasan

Pesimistiko sa kalagayan ng mga manggagawa na may himig nangungutya, nanunudyo, nanunumbat at naninisi, nagtatanong, nalilito sa mga kalagayan ng mga manggagawa sa lipunang ginagalawan. May halong pagka-optimistiko, hindi nawawalan ng pag-asa.

Kung Tuyo na ang Luha Mo, Aking Bayan

Ang Bayani

Ang Buhay
Nanunudyo ang persona sa kalagayan ng bansa sa kamay ng mga dayuhan at dito ay nagpapaalala sa malagim na kasaysayan ng bansa. May pagka- nasyonalismo ang tono dahil ipanaglalaban ang karapatan. Pesimistiko ang kabuuang tono sapagkat walang nakikitang pagbabago, halos pangit lahat ang nakikita sa persona ng kalagayan ng mga manggagawa.

Ipinapakita ang pagmamalaki sa ginagawa ng mga manggagawa. At mapanghamon at palaban dahil hindi natitinag sa mga pagsubok sa buhay. Ito ay pesimistiko sa pasalungat na paraan sa pagpapahayag tungkol sa mga manggagawa. pagkapesimistiko dahil sa kabiguan ng kanilang pangarap.

Optimistiko na may halong pesimistiko ang tinig ng persona sa tula. Optimistiko dahil naniniwala pa rin ang persona na hindi madadaig ang kabutihan sa kasamaan. Umaasa na mayroon pang darating pagbabago. Hindi niya nakalimutang banggitin ang Poong Maykapal. Pesimistiko sapagkat minsan ay nawawalan siya ng pag-asa bunga ng mga pangit na karanasan na kanyang nakikita. Paiba-iba minsan ang tono nito, may himig na nanunudyo, nanunumbat, naninisi at nagsusumbong. Mapangarap din ang kanyang tono at mapaghamon na gumawa ng hakbang para sa problemang nagbabadya. Gumagamit ang persona ng mga tayutay tulad ng pagtutulad, at pagwawangis, para maipahayag niya ang kaniyang hinanaing at damdamin.

Ang manggagawang pinag-uusapan sa tula ay isang Panday. Ang persona ay isang taong humahanga sa kanya. Optimistiko ang tono ng persona sa pagnanais na magbago sa sarili mula sa isang masama tungo sa isang mabuting mamamayan. Ang dating pangit na karanasan ayaw nang balikan. Ang manggagawang mambubukid ay may paninindigan. Inilarawan na ang panday ay isang masipag. Malilirip ito sa unang linya:

"Kaputol na bakal na galing sa bundok. Sa dila ng apoy kanyang pinalambot;"

Ang bakal ay nagpapakita ng katapangan at katigasan tulad ng isang tao na may sariling prinsipyo at pananaw sa buhay. Ang pagsusumikap na magbago nakatulong sa paggawa na galing sa kanyang puso at saloobin.

"Sa isang pandaya'y matyagang pinukpok, at pinagkahugis sa nasa ng loob”

Dahil sa sipag at tiyaga gamit ang kakayahan ay nabago at naging masaya ang buhay.

Pesimistiko ang kabuuang tono ng tula. Nag-iiwan ito ng katanungan. Diskontento sa kanilang kalagayan, pagtutuligsa sa mga pamamalakad ng kanilang amo, hindi maiintindihan at nalilito sa kalagayan ng pagawaan at sa kanilang kinalalagyan, na makikita sa linya na;

$$
\begin{gathered}
\text { "Bakit? Bakit } \\
\text { laging lupig } \\
\text { ang matuwid?" }
\end{gathered}
$$

Ang nasa katarungan ay inaapakan at palaging minamaliit ng ibang tao na may kaya. Palaging natatalo at 
nalulupig ang mga dukha sa mga mayayaman dahil pera ang pinapaandar at pinapagalaw. Nagsasaad din ito kung sino ang mga gumagawa ng mabuti sila ang mga masasama sa lipunan. Ang gumawa ng masama ay ang pinaniniwalaan sa lipunan. Napakalaking kabalintunaan ang pangyayari, na matutuhayan sa taludtod;

$$
\begin{gathered}
\text { “Di nasunod } \\
\text { pati Dios } \\
\text { na nag-utos. } \\
\text { Di tinupad, } \\
\text { binaligtad } \\
\text { pati batas." }
\end{gathered}
$$

Ang mga manggagawa na walang kalaban-laban ay pinagsamantalahan. Walang katiyakan ang kanilang kinalalagyan. Sa halip na ang pamahalaan at batas ang pumuprotekta ng mga manggagawa ngunit ang batas ay binabaligtad laban sa karapatan ng mga manggagawa. Ginawang pabor sa mga namumuhunan o kapitalista. Isang halimbawa ang kontrakwalisasyon. Ang mga mambabatas ay na iimpluwensyahan ng mga kapitalista dahil ang ibang mga kasamahan ay halos mga kapitalista din at namumuhunan.

Ang naging resulta, nagigipit ang mga manggagawa sa sitwasyon. Mas lalo silang nahihirapan at kawawa ang kanilang pamilya at mahal sa buhay na umaasa sa kanila. Isang katotohanan, kung sino ang lumilikha ng batas ay siya ring lumalabag.

\subsection{Ang patulang pananaw}

Ang pinakadiwa ng tula. Dito umiikot ang buong kaisipang tinatalakay ng persona. Kukunin ng mananaliksik ang mga tema o pananaw na gustong ipalabas ng tula na lumilitaw sa pagsusuri. Ang patulang pananaw na nangingibabaw sa tula ang kadakilaan ng mga manggagagawa na may malaking naiaambag sa lipunan.

\section{Talahanayan 2}

Ang patulang pananaw

\begin{tabular}{cl}
\hline Mga tula & Mga pananaw o tema \\
\hline Ang Panday & $\begin{array}{l}\text { Ang sinumang magnais na magbago kapag muling napukaw ang masaklap na nakaraan } \\
\text { ay muling magsisilab nakahimlay na poot. }\end{array}$
\end{tabular}

Ang Aklasan Ang karapatang mamuhay ng mapayapa at kalayaan sa pagkikilos nito.

Kung Tuyo na Ang Luha Mo, Ang nagmahal sa sariling lupa ay handang ipaglaban ito hangga't sa kamatayan. Aking Bayan

Ang Bayani Isang kabayanihan ninuman ang makagawa ng kabutihan lalo na kung ito'y ambag sa bayan.

Ang Buhay Ang buhay makahulugan kung ito’y binigyang ng pagpapahalaga.

Ang karaniwang tao kapag may trabaho sila, ang mga taong may naibabahagi sa lipunan. Ang kanilang lakas na inaalay nakakatulong sa kanyang kapwa tao. Bawat tao ay may karapatan na kailangan malaman para maipagtanggol ang sarili sa sinomang mang-aalipin. Ang katarungan na gustong makamit ay makamtan kung kusang ipaglalaban. Nagsisikap ang mga manggagawa sa kanilang iba’t ibang trabaho. Sa tula, may nagtatrabaho bilang magsasaka, empleyado ng pabrika, kargador, at tindera. Mayroon ding nagsisilbi bilang manglilitson, mananahi, karaniwang empleyado ng bangko, konstruksyon worker, at minero. Silang lahat ay may papel na ginagampanan sa buhay. Binubuhay ang pamilya sa pagtatrabaho. Naiahon ang mga namumuhunan o negosyo dahil sa kanilang kasipagan. Higit sa lahat, dahil sa kanila napaikot ng maayos ang ekonomiya ng bansa. Ngunit kabaligtaran ang lahat. Ipinamalas ng mga manggagawa na marunong silang masaktan at maghimagsik kapag nilulupig ng kanilang karapatan bagkus umiiral pa rin ang kalambutan ng kanilang puso at pagkabayani sa 
Bacalla, L. A.

anomang gawain.

\section{Talahanayan 3}

Ang pinag-uukulan

\begin{tabular}{ll}
\hline \multicolumn{1}{c}{ Mga tula } & \multicolumn{1}{c}{ Mga pinag-uukulan } \\
\hline Ang Panday & Ang mga taong nangmamaliit sa kakayahan ng manggagawa \\
Ang Aklasan & $\begin{array}{l}\text { Ang mga mamumuhunan, may-ari ng kompanya, may ari ng pabrika, sa mga } \\
\text { kapitalista, ang mga may-ari ng lupaing sinasakahan ng mga magsasaka at sa } \\
\text { pamahalaan o gobyerno. }\end{array}$
\end{tabular}

Kung Tuyo na Ang Luha Mo, Ang mga manggagawang inaalipin, sa mamamayang Pilipino at sa mga dayuhan. aking Bayan

Ang Bayani

Ang mga may-ari ng lupa, may-ari ng pabrika, kompanya ng minahan, may-ari ng komersyong gusali, sa pamahalaan at sa mga tagapangasiwa.

Ang Buhay

Sa mga namumuhunan, sa mga negosyante, sa mga kalakalan, sa pinaglilingkuran, at sa mga kapitalista.

Ang pinag-uukulang ng tula ay ang mga taong nagmamaliit sa mga manggagawa, sa pamahalaan, sa mga nang-aalipin, sa mga namumuhuan at mga may-ari ng kanilang pinagtratrabahuan. Sa pamamagitan ng linya ng tula ay naipaabot nila ang kanilang mensahe sa pamamagitan pinag-uukulan. Gamit ang mga matalinghagang salita sa pagpapahayag na nagpapahayag ng malalim na pagkakahulugan. Ang kahilang kahilingan ay namumutawi sa bawat linya ng tula. Ipinahiwatig na may mga taong hindi marunong tumanaw ng utang na loob sa mga mahihirap. Hindi nila iniisip na kung wala ang mga manggagawa hindi sila makakaranas ng kaluwagan sa buhay. Binabanggit sa tula ang may-ari sa lupang sinasakahan na kailangang bigyang malasakit ang mga magsasaka dahil sila ang nagpapahirap sa pananim. Isipin ng mga may-ari ng lupa na ang mga magsasaka ang nagpapatulo ng pawis, nagbibilad sa init at ulan para may masaganang ani ngunit konti ang kanilang nakukuha. Hindi sila nakatikim sa kanilang naaning palay dahil kulang ang kanilang natanggap na pamamahagi ng may-ari ng lupang sinasakahan. Hiling nito na tulungan ng mga inuukulan na makamtan ng mga manggagawa ang kanilang simpleng pangarap at ang pangarap sa buhay ng kanilang mga anak Matataguyod sa pinag-uukulan na pantay-pantay ang pagtrato ng mga manggagawa mahirap man o mayaman.

\section{Talahanayan 4}

Ang pinag-iisang daing

\begin{tabular}{|c|c|c|}
\hline $\begin{array}{l}\text { Ang pinag-iisang daing ng mga } \\
\text { manggagawa }\end{array}$ & Mga pananaw & Mga tula \\
\hline \multirow[t]{5}{*}{$\begin{array}{l}\text { Respetuhin ang karapatang pantao ng mga } \\
\text { manggagawa sa pamamagitan ng tamang sahod, } \\
\text { tamang benepisyo, tamang oras sa } \\
\text { pagtatrabaho, at kaligtasan sa trabaho. }\end{array}$} & $\begin{array}{l}\text { Ang sinumang magnais na } \\
\text { magbago kapag muling napukaw } \\
\text { ang masaklap na nakaraan ay } \\
\text { muling magsisilab, sa nakahimlay } \\
\text { na poot. }\end{array}$ & Ang Panday \\
\hline & $\begin{array}{l}\text { Ang karapatang mamuhay ng } \\
\text { mapayapa at kalayaan sa } \\
\text { pagkikilos nito. }\end{array}$ & Ang Aklasan \\
\hline & $\begin{array}{l}\text { Ang nagmahal sa sariling lupa ay } \\
\text { handang ipaglaban ito hangga't sa } \\
\text { kamatayan. }\end{array}$ & $\begin{array}{l}\text { Kung Tuyo na Ang Luha Mo, aking } \\
\text { Bayan }\end{array}$ \\
\hline & $\begin{array}{l}\text { Isang kabayanihan ninuman ang } \\
\text { makagawa ng kabutihan lalo na } \\
\text { kung ito'y ambag sa bayan. }\end{array}$ & Ang Bayani \\
\hline & $\begin{array}{l}\text { Ang buhay makahulugan kung } \\
\text { ito'y binigyan ng pagpapahalaga. }\end{array}$ & Ang Buhay \\
\hline
\end{tabular}


Ang pangkalahatang daing o hibik ng mga manggagawang Pilipino ay respetuhin ang karapatang pantao ng mga manggagawa sa pamamagitan ng tamang sahod, tamang benepisyo, tamang oras sa pagtatrabaho, at kaligtasan sa trabaho. Ito ay isa-isa nalilirip sa bawat tula una ang tulang panday na bigyan ang mga manggagawa ng tamang sahod at benepisyo. Siya ay nagsisipag hindi para sa kanya kung hindi para din sa iba;

“Ginawang araro: pangbuhay ng madla”

Sa tulang “Aklasan," ang mga magsasaka, empleyado ng pabrika, mga tindera, mga kargador, mga minero, at ibang uring manggagawa ay nagtatanong sa kanilang mahirap na sitwasyon;

$$
\begin{gathered}
\text { "Pagka't bakit } \\
\text { di kakain } \\
\text { ang nagtanim?" }
\end{gathered}
$$

Dahil nito, kumikilos sila upang madinig ang kanilang tinig;

$$
\text { “Welga! Welga!” }
$$

Sa tulang "Kung Tuyo na ang Luha Mo, Aking Bayan," ang titukoy na mga manggagawa ay iyong katulad ni Huli at Sisa na naaliping bayad -utang at binaliw ng kahirapan. Silang lahat ay nanunudyo;

\section{"Katulad mo ay si Huli, naaliping bayad-utang," \\ Katulad mo ay si Sisa, binaliw ng kahirapan;}

Ang mga magsasaka, minero, mikaniko, at konstruksyon workers sa tulang “Ang Bayani," ay sumisigaw na ang mga manggagawa ay bigyan ng tamang pasahod ang kanilang paghihirap sa pagtatrabaho;

$$
\begin{gathered}
\text { “Sambundok na ginto ang aking dinungkal, } \\
\text { kahit na kaputol, di binahaginan! } \\
\text { ang aking inani’t sambukiring palay, } \\
\text { ngunit wala akong isaing man lamang " }
\end{gathered}
$$

At sa tulang "Ang Buhay“, ang sigaw ng mga manggagawa ay maiahon ang kanilang katayuan sa buhay;

$$
\begin{gathered}
\text { "Habang nagsisikap } \\
\text { at nag-aadhika, } \\
\text { dahil sa pangarap na ligaya't tuwa, } \\
\text { lalong sinisiil ng pagdaralita." }
\end{gathered}
$$

Ang ibang kabuoang pinag-isang daing ng mga manggagawa ay bigyan sila ng tamang oras at kaligtasan sa pagtatrabaho. Ipinakita sa mga piling tula na mataas ang oras ng mga magsasaka, empleyado ng pabrika, mga tindera, mga kargador, at mga minero sa pagtatrabaho. Mapanganib din sa kanilang kalusugan. Ang magsasaka at minero ay bumubungkal ng lupa na nangangailangan ng lakas at pawis. Ang empleyado ng pabrika at tindera ay tumayong maghapon. Ang mga kargador ay mapanganib din ang kanilang trabaho na pinapasan ang mga mabibigat na bagay na maaaring ikasawi sa kanilang buhay. Pinasikat sa gabi ang araw sa tulang "Ang Bayani”. At sa "Ang Buhay", ang lahat ng mga magaganda ay may wakas. Ang mananatili ay ang pangarap ng mga manggagawa. Sa pagtatrabaho bigyan halaga ang kaligtasan dahil mahirap at mapanganib ang trabaho ng mga karaniwang manggagawa. Ang kanilang trabaho ay mahirap at mabigat kaya bigyang diin ang kanilang kalusugan at kaligtasan.

Inilarawan ang kalagayan ng mga manggagawa panahon ng Kastila at Amerikano. Magsasaka, minero, mekaniko, konstruksyon worker ang binabanggit sa tulang “Ang Bayani”. Mapanganib ang kanilang kalusugan. At sa kabila nito sila ay patuloy na nangangarap sa kasaganaan at kaginhawaan sa pamumuhay. Ang lahat ng kaguluhan sa paggawaan ay may lunas bunga ng kanilang pagsasakripisyo sa pagtatrabaho. Makakamtan ang 
Bacalla, L. A.

katahimikan kung maiipapatupad at respetuhin ang isinasaad sa Saligang Batas, Artikulo XIII, sek.3. talata 4, qtd in Cruz 439: "The State shall regulate the relations between workers and employees, recognizing the rights of labor to its just share in the fruits of production and the right of enterprises to reason-able returns and investment and expansion and growth. Sa pamamagitan ng Saligang Batas, maprotektahan ang mga karapatan ng mga manggagawa at mga negosyante at makamtan ang kapayapaan.

\section{Konklusyon at rekomendasyon}

Batay sa mga natuklasan, ang konklusyong nabuo sa mga piling tula ni Amado Vera Hernandez ay nagsisiwalat sa mga hibik ng mga manggagawa sa lipunan gamit ang persona o nagsasalita, pinag-uukulan, sa patulang pananaw, imahe, at sa paggamit tayutay.Ito ay naglalantad na ang panitikan ay sumasalim sa tunay na buhay. Sa tinig ng tula bawat manggagawang Pilipinong ay nangangailang ng suporta mula sa pamahalaan. Sa Patulang pananaw ay napapahalagahan ang kabayanihan ng mga manggagawa sa lipunan. Hangad ng manggagawa na magbibigyan ang kanilang pangkahatang hiling.

Inirerekomenda na magsagawa ng ibayong pag-aaral sa ibang genre ng panitikan na tumatalakay sa isyung panlipunan. Mahikayat ang mga guro na magsasagawa ng panunuring pampanitikan kasakuyang umiiral sa isyu. Pagyamanin ang pinag-isang daing ng mga manggagawa sa pamamagitan ng pagdaraos ng seminar at mag-anyaya ng kawani ng Department of Labor and Employment (DOLE) na ang tema ay "Karapatan ng mga Manggagawa at mga Tagapangasiwa" upang malaman ang limitasyon ng bawat panig.

\section{Mga Sanggunian}

Azucena, C. A. (1996). The labor code with comments and cases (Vol. 2). Rex Bookstore.

Cericos, E. U. (2003). Analysis of literary validities [Pagsusuri sa mga bisang pampanitikan ng tula]. [Dissertation].

Cruz, I. A. (2007). Constitutional law. Central Book Supply.

Hechanova, S., \& Rebadomia, M. (2010). Political economic analysis of the plight of farm workers in Hacienda Bolo-Bolo and Hacienda Sta. Maria Negros Occidental [Pampulitikang ekonomiyang pagsusuri sa kalagayan ng mga manggagawang bukid sa Hacienda Bolo-Bolo at Hacienda Sta. Maria Negros Occidental]. http://dspace.cas.upm.edu.ph:8080/jspui/handle/123456789/349

Ortiz, W. (2008). Children on the sidelines: The violence and ignorance of the space of young workers [Mga batang sagigilid: Ang dahas at dalumat ng espasyo ng mga batang manggagawa]. Malay Journal, 21(6), 27-41. https://doi.org/10.3860/malay.v21i1.687

Pante, M. D. (2013). Modern urban transport workers [Mga modernong manggagawa ng transportasyong panlungsod]. Malay Journal, 25(2), 21-35.

San Juan, D. (2018). National salvage and chain of defense: A critical analysis of the Philippine Labor Export Policy [Pambansang salbida at kadena ng depensiya: Isang kritikal na pagsusuri sa Labor Export Policy ng Pilipinas]. Tanggol Wika.

Sedigo, E. J. (2003). Environmental values contained in Filipino poems: An analysis [Mga pagpapahalaga sa kapaligiran na napapaloob sa mga tulang Filipino: Isang pagsusuri]. Cebu Normal University.

Villafuerte, P. V. (2000). Literary analysis [Panunuring pampanitikan]. Mega Jesta Prints.

Wong, R. L. (2008). The metaphysical of William Wordsworth [Thesis, Cebu Normal University].

Yu, R. T. (2006). Kilates. Literary analysis [Kilates: Panunuring pampanitikan]. C \& E Publishing. 